\title{
Model Identification and Course Controller Design for Unmanned Surface Vehicle
}

\author{
Dongdong Mu, Guofeng Wang*, Yunsheng Fan and Yongsheng Zhao \\ School of Information Science and Technology, University of Dalian Maritime \\ Dalian, Liaoning, 116026, China \\ mu_dong@yeah.net; gfwangsh@163.com; yunsheng@dlmu.edu.cn
}

\begin{abstract}
This paper addresses two interrelated problems concerning the course control of unmanned surface vehicle (USV), namely, the model identification and the course controller design. First of all, field experiments (turning test and zig-zag test) are carried out to collect experimental data, then recursive least squares and data fitting methods are used to identify the parameters of the Norrbin model. Considering the uncertainty of the model, the method of non singular terminal sliding mode (NTSM) is employed to design course controller. Meanwhile, in order to reduce control gain, disturbance observer $(D O B)$ is used to compensate the external disturbance, and DOB-NTSM course controller is proposed. But the inherent characteristic of NTSM can lead to chattering of control input, RBF neural network (NN) is employed to reduce the chattering phenomenon. Finally, classical PID and NTSM control law are compared with the DOB-NTSM. Simulation results show that DOB-NTSM course controller has good control effect, which proves its correctness and feasibility.
\end{abstract}

Keywords: USV, Model Identification, Course Control, NTSM, DOB, RBF

\section{Introduction}

With the continuous progress of science and technology, various marine exploration devices are developed. As an intelligent equipment, USV is mainly used to perform dangerous and unsuitable personnel operating tasks [1]. The design of course controller is an important research topic of ship motion control, because it is not only related to the economy and security navigation but also the foundation of automatic collision avoidance, trajectory tracking and ship stabilization. In the process of studying the course control, the mathematical model of USV is essential. So how to obtain the model parameters of USV is an very important question.

Recalling previous papers, many excellent papers and algorithms are used to solve the problem of model identification. Professor Guedes's research team in Portugal Soares uses extended Kalman filter [2] and genetic algorithm [3] to identify the hydrodynamic coefficients of the nonlinear response model and the hydrodynamic coefficients of integral mode. In [4], for a high speed trimaran, on the basis of known parameters, the unknown parameters are identified, and then unscented Kalman filter method is used to optimize the previous identification results. In [5], particle swarm algorithm is replaced by a fast interior point method to identify a three degree freedom model of a small unmanned boat with twin propeller and twin rudder. Paper [6] describes planar motion model for an USV, and a three degree freedom model is obtained by the method of system identification on the basis of considering the sideslip angle. The above papers have obtained more accurate ship model parameters, but their methods are relatively complex. So in this paper, a simple identification scheme is proposed to identify the model parameters and the

Received (December 7, 2016), Review Result (January 13, 2017), Accepted (February 8, 2017) 
authenticity of the identification results are proved in Section 2.4. On the basis of identifying the model of USV, course controller is designed. Paper [7] proposes a controller for pod-driven surface ship based on adaptive backstepping with integrator. Paper [8] uses RBF NN to approach the part which is difficult to calculate of the control law. By combining the advantages of the robustness of PID and fast learning speed of wavelet network, a wavelet-network-based PID course controller is proposed in [9]. In this paper, on the basis of considering the model uncertainty, NTSM is employed to design course controller.

The advantages of this paper lie in that it presents a complete set of programs to identify the model parameters of USV and the method of identification is simple and easy to implement. In addition, considering the influence of model uncertainty and external disturbance, NTSM is employed to design course controller. At the same time, DOB is designed to compensate disturbance and RBF NN is used to weaken the chattering of the sign function.

The rest of the paper is organized as follows. Section 2 introduces the field experiments and model identification. In Section 3, DOB-NTSM course controller is designed. In Section 4, numerical simulations are carried out to show the rapidity and robustness of our design. Finally, some conclusions are made and future research directions are introduced in Section 5.

\section{USV Model Identification}

In this paper, field experiments and course controller design are based on the Dalian Maritime University "LANXIN" USV [10]. "LANXIN" USV is an intelligent platform which has many advanced equipments such as AIS, DGPS, far infrared and so on. It is shown in Figure 1.

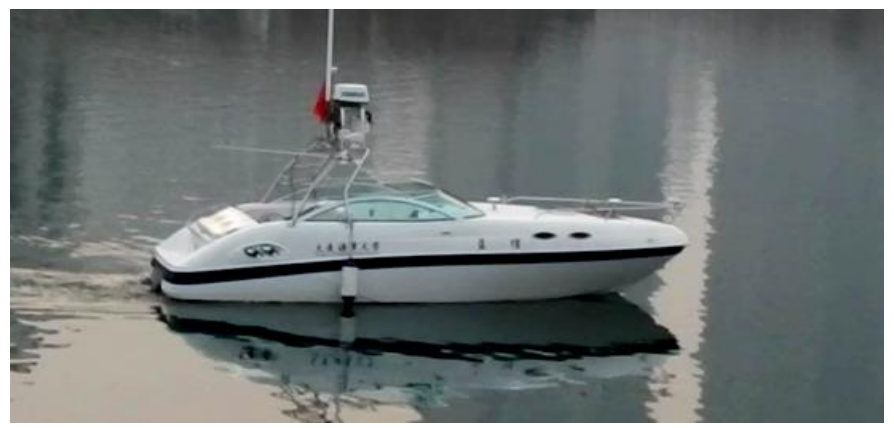

Figure 1. LANXIN USV

\subsection{USV Model and Identification Method}

Norrbin model [11] is selected to describe USV. It can show the relationship between rudder angle $\delta$ and course $\psi$ excellently. Norrbin model is shown as formula (1).

$$
\mathrm{T} \ddot{\psi}+\alpha \dot{\psi}^{3}+\dot{\psi}=K \delta
$$

$\mathrm{K}, \mathrm{T}$ and $\alpha$ are the model parameters. The essence of Norrbin model can be regarded as a linear Nomoto model with a nonlinear term $\alpha$. The model of Nomoto is shown as formula (2).

$$
\mathrm{T} \ddot{\psi}+\dot{\psi}=K \delta
$$

In this paper, recursive least squares (RLS) is applied to identify the parameters of Nomoto model. RLS is shown as formula (3). 


$$
\left\{\begin{array}{l}
\hat{\theta}(n)=\hat{\theta}(n-1)+K(n)\left[y(n)-\phi^{T}(n) \hat{\theta}(n-1)\right] \\
K(n)=\frac{P(n-1) \phi(n)}{1+\phi^{T}(n) P(n-1) \phi(n)} \\
P(n)=\left[I-K(n) \phi^{T}(n)\right] P(n-1)
\end{array}\right.
$$

$\hat{\theta}$ is the estimation value of the ship, and $\phi(k)$ is the data vector [12].

\subsection{Field Experiment}

In this section, field experiments are carried out to collect corresponding data to identify the model parameters. In order to obtain more accurate data, this experiment address is selected in the Dalian bridge outside, and the condition of the sea is relatively stable. The experiment address is shown in Figure 2.

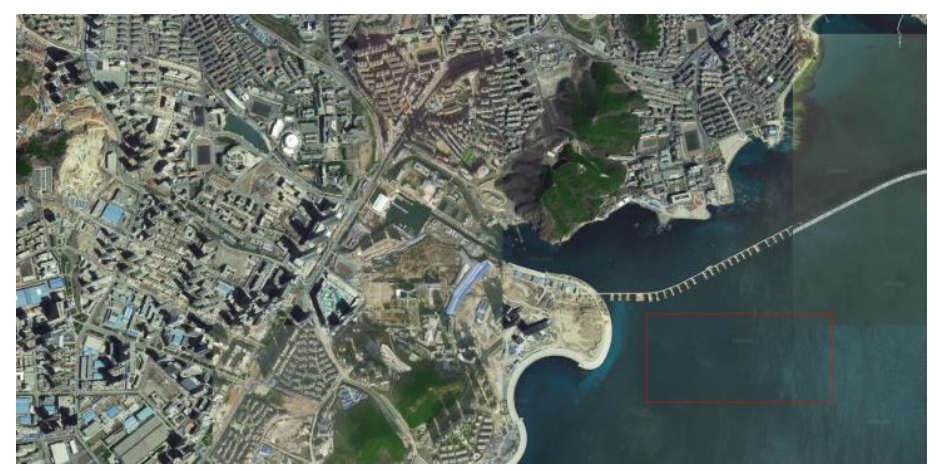

Figure 2. Experiment Address

According to the international practice, field experiments include turning test and zigzag test. Under the condition of keeping speed (about 9 knots) unchanged, field experiments are carried out.

Experimental contents:

(1) Turning test of $5^{\circ}, 8^{\circ}, 12^{\circ}, 15^{\circ}, 17^{\circ}, 18^{\circ}$ and $20^{\circ}$ are carried out.

(2) Zig-zag test of $15^{\circ} / 15^{\circ}$ is carried out.

The host computer records the data once every 0.5 seconds. The recorded data are very much, so we only list a portion of zig-zag test data in Table 1 . Theoretically speaking, for turning test, each constant value of rudder angle $\delta$ corresponds to a constant value of yaw rate $r$, but because of the existence of external disturbance, we can only seek an average value of yaw rate $r$. The corresponding data is shown in Table 2 .

Table 1. Zig-zag Test Data

\begin{tabular}{|c|c|c|}
\hline Marker & Rudder Angle & Course \\
\hline 1 & $-12.4^{\circ}$ & $235.04^{\circ}$ \\
\hline 2 & $-16.9^{\circ}$ & $224.64^{\circ}$ \\
\hline 3 & $-17.4^{\circ}$ & $220.78^{\circ}$ \\
\hline$\cdots$ & $\cdots$ & $\cdots$ \\
\hline 28 & $11.9^{\circ}$ & $288.61^{\circ}$ \\
\hline 29 & $13.3^{\circ}$ & $232.33^{\circ}$ \\
\hline 30 & $13.3^{\circ}$ & $237.52^{\circ}$ \\
\hline
\end{tabular}




\section{Table 2. Rudder Angle and Yaw Rate}

\begin{tabular}{|c|c|c|}
\hline Marker & Rudder Angle & $r$ \\
\hline 1 & $5^{\circ}$ & 3.38 \\
\hline 2 & $8^{\circ}$ & 6.261 \\
\hline 3 & $12^{\circ}$ & 7.543 \\
\hline 4 & $15^{\circ}$ & 8.832 \\
\hline 5 & $17^{\circ}$ & 9.421 \\
\hline 6 & $18^{\circ}$ & 9.811 \\
\hline 7 & $20^{\circ}$ & 11.201 \\
\hline
\end{tabular}

\subsection{Model Identification}

Nomoto model is identified by zia-zag test data. The identification curves are shown in Figure 3. $a_{1} \sim a_{3}$ and $b_{1} \sim b_{3}$ are the coefficients of discrete transfer function. The transfer function is

$$
\frac{0.2743 z^{2}+0.2122 z-0.118}{z^{3}-0.9537 z^{2}-0.5476 z+0.007182}
$$

The next step is changing (4) into a continuous transfer function (5).

$$
\frac{1.65 s^{3}-10.63 s^{2}+88.19 s+225.9}{s^{4}+14.26 s^{3}+106.8 s^{2}+322 s-0.7904}
$$

Due to the coefficients of higher order and low order are differ greatly, so (5) can be simplified as (6).

$$
\frac{225.9}{106.8 s^{2}+332 s}
$$

The formula (6) is converted to a differential formula and the result is compared with (2). Then we can get that $K=0.707, T=0.332$.

$\alpha$ is fitted by turning test data. When the turning test is carried out, $\dot{r}, \ddot{r}$ and $\ddot{\delta}$ are equal to zero. So formula (1) can be simplified as

$$
\alpha \dot{\psi}^{3}+\dot{\psi}=K \delta
$$

As can be seen from formula (7), due to $\delta$ and $r$ are one to one, so we can regard $r$ as input and $\delta$ as output. The fitting curve is shown in Figure 4. We can get that $\alpha=0.001$.

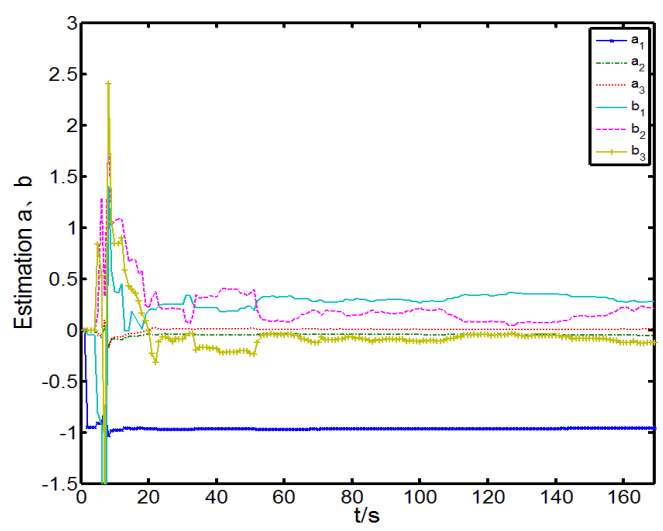

Figure 3. Identification Curves 


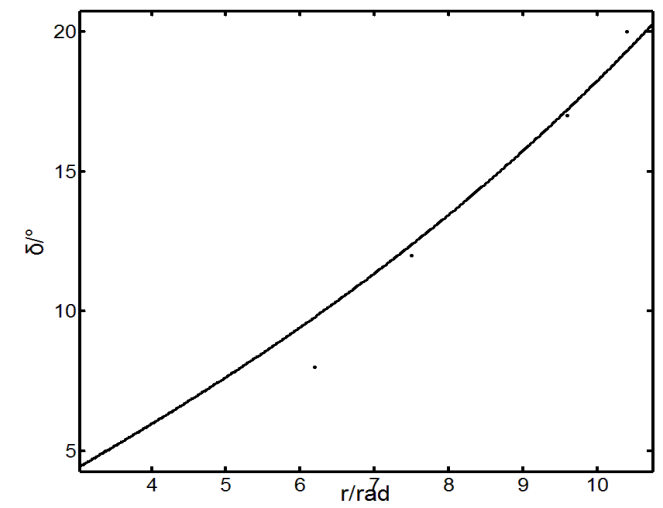

Figure 4. Fitting Curve

\subsection{Model Validation}

Due to the characteristic of rudder is not considered in this paper, so only turning test is hired to verify the correctness of the identification results. The actual turning test of $5^{\circ}$ curve is shown in Figure 5. The simulation turning test of $5^{\circ}$ curve is shown in Figure 6.

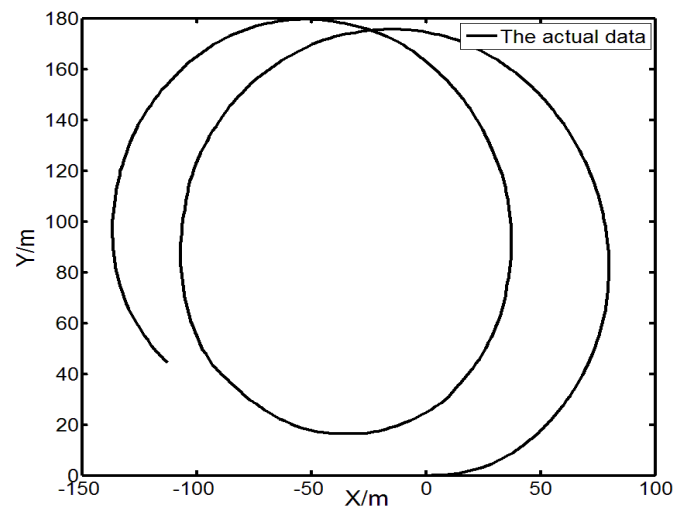

Figure 5. The Actual Turning Test

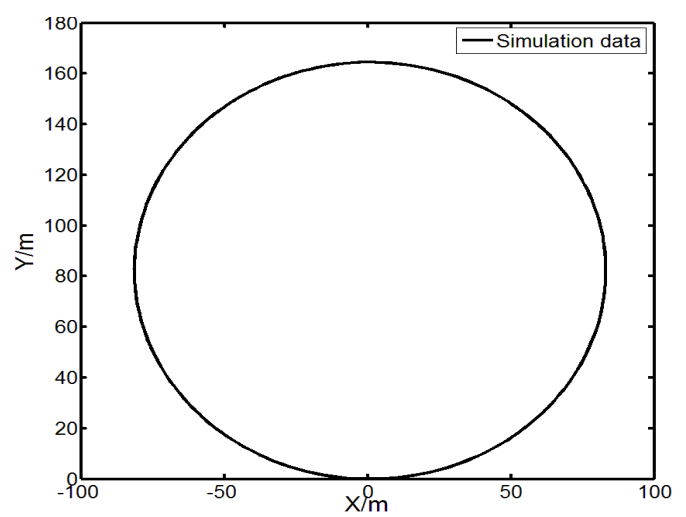

Figure 6. The Simulation Turning Test

Although we have chosen a relatively calm sea to carry out field experiments, external disturbance still affects the experiment results. From Figure 5 we can see that the curve of turning test appears drift, and the actual radius of gyration is $84.9716 \mathrm{~m}$. From Figure6 we can see that the simulation radius of gyration is $85.7621 \mathrm{~m}$. The error of the two is very small, so it is proved that the identification result is correct. 


\section{Course Control Design}

\subsection{Problem Formulation}

Select state variable $x_{1}=\psi, x_{2}=\dot{\psi}=r$ and $u=\delta$, so formula (1) can be simplified as

$$
\left\{\begin{array}{l}
\dot{x}_{1}=x_{2} \\
\dot{x}_{2}=f(x)+b u+g(t) \\
y=x_{1}
\end{array}\right.
$$

Where $f(x)=-\frac{1}{T} x_{2}-\frac{\alpha}{T} x_{2}^{3}, b=\frac{K}{T}, u \in R$ and $y \in R$ are the input and output of the system; $g(t)$ is the total disturbance of system (uncertainty of model and external disturbance), $\|g(t)\|<l_{g}, l_{g}>0$.

Control objective: Design control input $u$ to make the course $\psi$ follow the target course $x_{d}$.

Assumption 1. The reference course $x_{d}$ is a sufficiently smooth function of time, and $x_{d}, \dot{x}_{d}, \ddot{x}_{d}$ are bounded.

Assumption 2. $g(t)$ is slow change, that is to say that $\dot{g}(t)=0$.

\subsection{Non Singular Terminal Sliding Mode}

Sliding mode control is a kind of special nonlinear control method, because of its good robustness and simple physical realization, it has been widely used in the control of all kinds of nonlinear systems [13]. Based on the sliding mode control, the terminal sliding mode is proposed in the paper [14], and it is widely used in the control of mechanical arm. Terminal sliding mode has a fatal shortcoming that in some particular cases, the system is singular, causing the system to crash [15]. In order to overcome the singularity problem of terminal sliding mode, non-singular terminal sliding mode is proposed paper [16]. It not only overcomes the singular problem but also achieves the system state of the finite time to reach, and obtain a higher convergence accuracy. In this paper, NTSM is employed to design the course controller.

Define tracking error $e_{1}=x_{1}-x_{d}$ and $e_{2}=\dot{x}_{1}-\dot{x}_{d}$, then the switching function of NTSM is defined as

$$
s=e_{1}+\frac{1}{\lambda} e_{2}^{\frac{p}{q}}
$$

Where $\lambda$ is normal number, $q_{2}$ and $p_{2}$ are odd numbers, and $p>q, 1<p / q<2$.

The control law $u$ is designed as

$$
u=-b^{-1}\left[f(x)+\lambda \frac{q}{p} e_{2}^{2-\frac{p}{q}}+\left(l_{g}+\eta_{1}\right) \operatorname{sgn}(s)-\ddot{x}_{d}\right]
$$

$\eta_{1}$ is a constant value greater than zero. Then the time derivative of formula (9).

$$
\dot{s}=s\left(e_{2}+\frac{1}{\lambda} \frac{p}{q} e_{2}^{\frac{p}{q}-1} \dot{e}_{2}\right)=e_{2}+\frac{1}{\lambda} \frac{p}{q} e_{2}^{\frac{p}{q}-1}\left(f(x)+b u+g(t)-\ddot{x}_{d}\right)
$$

Define $\phi\left(e_{2}\right)=\frac{1}{\lambda} \frac{p}{q} e_{2}^{\frac{p}{q}-1}$ 
Then

$$
\dot{s}=e_{2}+\phi_{1}\left(e_{2}\right)\left(f(x)+b u+g(t)-\ddot{x}_{d}\right)
$$

In order to verify the stability of the proposed algorithm, the first Lyapunov function is selected.

$$
V_{1}=\frac{1}{2} s^{2}
$$

Then the time derivative of $V_{1}$ along the solution of (10) and (12) is

$$
\begin{aligned}
& \dot{V}_{1}=s \dot{s} \\
& =s\left[e_{2}+\phi\left(e_{2}\right)\left(f(x)+b u+g(t)-\ddot{x}_{d}\right)\right] \\
& =s\left[e_{2}+\phi\left(e_{2}\right)\left(-\lambda \frac{q}{p} e_{2}^{2-\frac{p}{q}}-\left(l_{g}+\eta_{1}\right) \operatorname{sgn}(s)+g(t)\right)\right] \\
& =s \phi\left(e_{2}\right)\left(-\left(l_{g}+\eta_{1}\right) \operatorname{sgn}(s)+g(t)\right) \\
& \leq-\phi\left(e_{2}\right) \eta_{1}|s|
\end{aligned}
$$

So far, we can know that $\dot{V}_{1} \leq 0$, and the system is stable.

\subsection{DOB}

If the total disturbance $g(t)$ increases, we can maintain the robustness of the system by increasing the value of $l_{g}$. But this will aggravate system chattering, and it is not conducive to the stability of the system. Besides, in the above analysis, we assume that the total disturbance $g(t)$ bound is known, but in reality, it is difficult to know the bound of $g(t)$. In order to solve this problem, DOB is designed in this paper.

$$
\left\{\begin{array}{l}
\dot{\hat{x}}_{2}=f(x)+\hat{g}(t)+b u+k_{1} \tilde{x}_{2} \\
\dot{\hat{g}}(t)=k_{2} \tilde{x}_{2}
\end{array}\right.
$$

Where $k_{1}$ and $k_{2}$ are positive definite parameters. $\hat{g}(t)$ is the estimated value of $g(t)$ and $\hat{x}_{2}$ is the estimated value of $x_{2} \cdot \tilde{x}_{2}=x_{2}-\hat{x}_{2}$ and $\tilde{g}(t)=g(t)-\hat{g}(t)$.

So the control law (10) becomes

$$
u=-b^{-1}\left[f(x)+\lambda \frac{q}{p} e_{2}^{2-\frac{p}{q}}+\hat{g}(t)+\eta_{2} \operatorname{sgn}(s)-\ddot{x}_{d}\right]
$$

In order to verify the stability, the second Lyapunov function is selected.

$$
V_{2}=\frac{1}{2} s^{2}+\frac{1}{2 k_{2}} \tilde{g}(t)^{2}+\frac{1}{2} \tilde{x}_{2}^{2}
$$

Then the time derivative of $V_{2}$ along the solution of (14) is

$$
\begin{aligned}
& \dot{V}_{2}=s \dot{s}+\frac{1}{k_{2}} \tilde{g}(t) \dot{\tilde{g}}(t)+\tilde{x}_{2} \dot{\tilde{x}}_{2} \\
& =s \phi\left(e_{2}\right)\left[g(t)-\hat{g}(t)-\eta_{2} \operatorname{sgn}(s)\right]+\frac{1}{k_{2}} \tilde{g}(t)(\dot{g}(t)-\dot{\hat{g}}(t))+\tilde{x}_{2}\left(\dot{x}_{2}-\dot{\hat{x}}_{2}\right) \\
& =s \phi\left(e_{2}\right)\left[\tilde{g}(t)-\eta_{2} \operatorname{sgn}(s)\right]-\tilde{g}(t) \tilde{x}_{2}+\tilde{x}_{2}\left(\tilde{g}(t)-k_{1} \tilde{x}_{2}\right) \\
& =s \phi\left(e_{2}\right)\left[\tilde{g}(t)-\eta_{2} \operatorname{sgn}(s)\right]-k_{1} \tilde{x}_{2}^{2} \\
& \leq-\phi\left(e_{2}\right)\left(\eta_{2}-\tilde{g}(t)\right)|s|-k_{1} \tilde{x}_{2}^{2}
\end{aligned}
$$


Define $\eta_{2}=|\tilde{g}(t)|+\xi(\xi>0)$ and $\phi\left(e_{2}\right)>0$, then $\dot{V}_{2} \leq-\phi\left(e_{2}\right) \xi|s|-k_{1} \tilde{x}_{2}^{2} \leq 0$.

\subsection{Reduce Chattering}

The sign function will result in the chattering of the control quantity and it is not conducive to the stability of the system. So in order to solve this problem, RBF NN [17$18]$ is used to estimate sign function. RBF NN can be described as $W^{T} \phi(x)$ with weight vector $W \in R^{q_{1}}$, note number $q_{1}$, input vector $\mathrm{x} \in \Omega \in R^{n}$ and basis function vector $\phi(x) \in R^{q_{1}}$. In the existing literature [19], universal approximation results indicate that if $q_{1}$ is chosen to be sufficiently large, then $W^{T} \phi(x)$ can be approximated any nonlinear function. The network structure is shown in Figure 7.

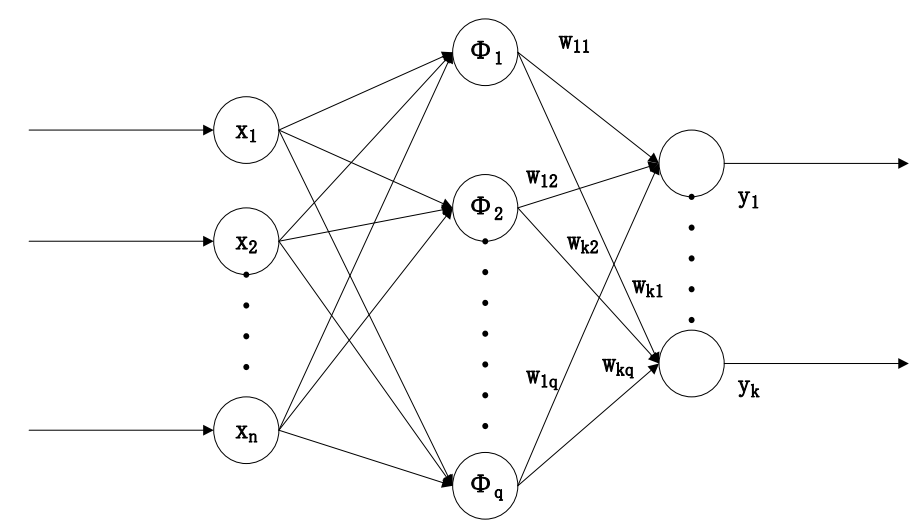

Figure 7. RBF NN Structure

Define $\operatorname{sgn}(s)=Q(x), \operatorname{RBF} \mathrm{NN}$ is used to approximate function $Q(x)$

$$
\hat{Q}(x)=W^{T} \phi(x)
$$

Where the weight vector $W=\left[w_{1}, w_{2}, \ldots, w_{q}\right]^{T} \in R^{l}$, the NN node number $l>1$ and $\phi(x)=\left[\phi_{1}(x), \phi_{2}(x), \ldots \phi_{q_{1}}(x)\right]^{T}$, with $\phi_{i}(x)$ being chosen as the commonly used Gaussian function, which have the from

$$
\phi_{i}(x)=\exp \left[-\frac{\left(x-c_{i}\right)^{T}\left(x-c_{i}\right)}{2 \sigma_{i}^{2}}\right], i=1,2, \ldots, q_{1}
$$

Where $\mathrm{x}=\left[x_{1}, x_{2}, \ldots, x_{n}\right]$ is the input samples, $c_{i}=\left[c_{i 1}, c_{i 2}, \ldots ., c_{i n}\right]$ is the center of the receptive field and $\sigma_{\mathrm{i}}$ is the width of the Gaussian function. The output of the network can be described as

$$
y_{k}=\sum_{i=1}^{q} w_{k i} \phi_{i}\left(\left\|x-c_{i}\right\|^{2}\right), i=1,2,3, \ldots, q_{1}
$$

Where $y_{k}$ is the output of the $k$ node of the output layer. RBF NN can approximate any function $Q(x)$ over a compact set $\Omega_{x} \subset R^{n}$ to any arbitrary accuracy as

$$
\begin{aligned}
& Q(x)=W^{* T} \phi(x)+\varepsilon \quad \forall x \subset \Omega_{x} \\
& W^{*}:=\arg \min _{W \subset R^{q \mid}}\left\{\sup _{x \subset \Omega_{x}}\left|f(x)-W^{T} \phi(x)\right|\right\}
\end{aligned}
$$


Where $W^{*}$ is the ideal constant weights and $\varepsilon$ is the approximation error. The ideal constant weights vector $W^{*}$ and function approximation error $\varepsilon$ of NNs are bounded by

$$
\left\|W^{*}\right\| \leq W_{\mathrm{m}},|\varepsilon| \leq \varepsilon_{\mathrm{m}} \quad \forall x \subset \Omega_{x}
$$

Where $W_{m}$ and $\varepsilon_{m}$ are positive constants. In this paper, define $x=s, \sigma=5$ and $l=40$.

So control law (14) can become

$$
u=-b^{-1}\left[f(x)+\lambda \frac{q}{p} e^{2-\frac{p}{q}}+\hat{g}(t)+\eta_{3} \hat{Q}(x)-\ddot{x}_{d}\right]
$$

Define

$$
\dot{\hat{W}}=\gamma s \phi\left(e_{2}\right) h(x)
$$

where $\gamma$ is a design parameter and its greater than zero. $\hat{W}$ is the estimated value of $W . \tilde{W}=W-\hat{W}$ and $\tilde{Q}(x)=Q(x)-\hat{Q}(x)$.

So we can get

$$
\begin{aligned}
& \dot{s}=e_{2}+\phi\left(e_{2}\right)\left(f(x)+b u+g(t)-\ddot{x}_{d}\right) \\
& =\phi\left(e_{2}\right)(-\hat{Q}(x)+Q(x)-Q(x)+g(t)-\hat{g}(t)) \\
& \left.=\phi\left(e_{2}\right)\left(\tilde{W}^{T} \phi(x)-Q(x)+g(t)-\hat{g}(t)+\varepsilon\right)\right)
\end{aligned}
$$

Define the third Lyapunov function.

$$
V_{3}=\frac{1}{2} s^{2}+\frac{1}{2 \gamma} \tilde{W}^{T} \tilde{W}+\frac{1}{2 k_{2}} \tilde{g}(t)^{2}+\frac{1}{2} \tilde{x}_{2}^{2}
$$

Then the time derivative of $V_{3}$ along the solution of (22) and (23) is

$\dot{V}_{3}=s \dot{s}-\frac{1}{\gamma} \tilde{W}^{T} \dot{\hat{W}}+\frac{1}{k_{2}} \tilde{g}(t) \dot{\tilde{g}}(t)+\tilde{x}_{2} \dot{\tilde{x}}_{2}$

$=s \phi\left(e_{2}\right) \tilde{W}^{T} \phi(x)-\frac{1}{\gamma} \tilde{W}^{T} \dot{\hat{W}}+s \phi\left(e_{2}\right)(-Q(x)+g(t)-\hat{g}(t)+\varepsilon)+\frac{1}{k_{2}} \tilde{g}(t) \dot{\tilde{g}}(t)+\tilde{x}_{2} \dot{\tilde{x}}_{2}$

$\leq \phi\left(e_{2}\right)\left(s \varepsilon+s \tilde{g}(t)-\eta_{3}|s|\right)-\tilde{g}(t) \tilde{x}_{2}+\tilde{x}_{2}\left(g(t)-\hat{g}(t)-k_{1} \tilde{x}_{2}\right)$

$\leq-\phi\left(e_{2}\right)|s|\left(\eta_{3}-\tilde{g}(t)-\varepsilon\right)-k_{1} \tilde{x}_{2}^{2}$

Define $\eta_{3}=|\tilde{g}(t)|+\varepsilon_{\mathrm{m}}+\xi(\xi>0)$ and $\phi\left(e_{2}\right)>0$, then $\dot{V}_{3} \leq-\phi\left(e_{2}\right) \xi|s|-k_{1} \tilde{x}_{2}^{2} \leq 0$.

Theoretical results on stability analysis indicate that our proposed controller guarantees uniformly ultimate boundedness of all signals of the closed-loop system.

\section{Numerical Simulations}

In order to prove the correctness of the control strategy, classical PID controller and control law (10) are compared with DOB-NTSM course controller. The control law of PID is

$$
u=K_{p} e_{1}+K_{i} \int_{0}^{t} e_{1} d \tau+K_{d} \dot{e}_{1}
$$

Where $K_{p}, K_{i}, K_{d}$ are the parameters of PID and $K_{\mathrm{p}}=0.72, K_{i}=0.001$ and $K_{d}=0.6$. For control law (10), $\lambda=1.3, p=11, q=9, l_{g}+\eta_{1}=7.2$. For control law (14), $\eta_{3}=1, \gamma=1.1, k_{1}=1, k_{2}=10$. In order to show the role of DOB, assume that the 
uncertainty of model is $\sin (0.4 t)$, and the external disturbance is $2.5 \sin (0.6 t)$. This is to say $g(t)=2.5 \sin (0.6 t)+\sin (0.4 t)$.

The results of numerical simulations are shown in Figures. 8-11.

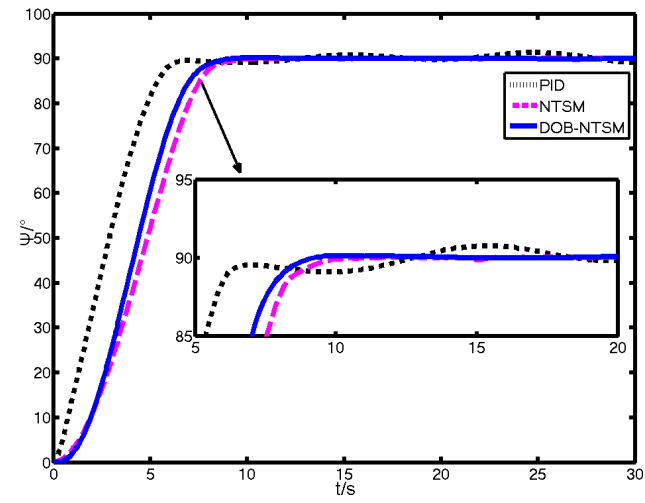

Figure 8. Course Curves

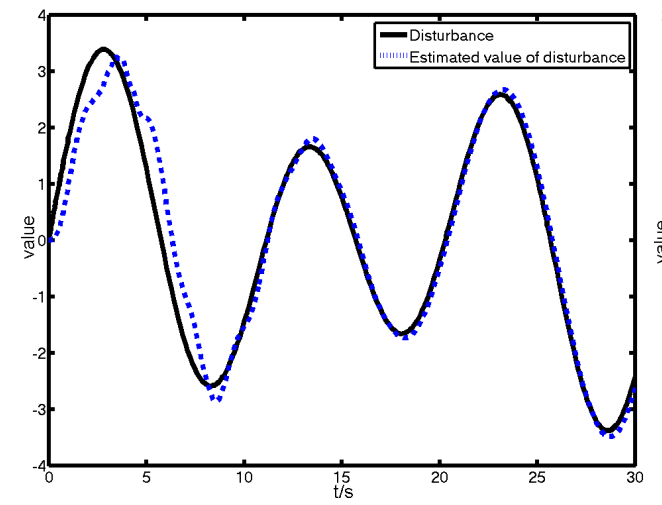

Figure 10. Disturbance Curves

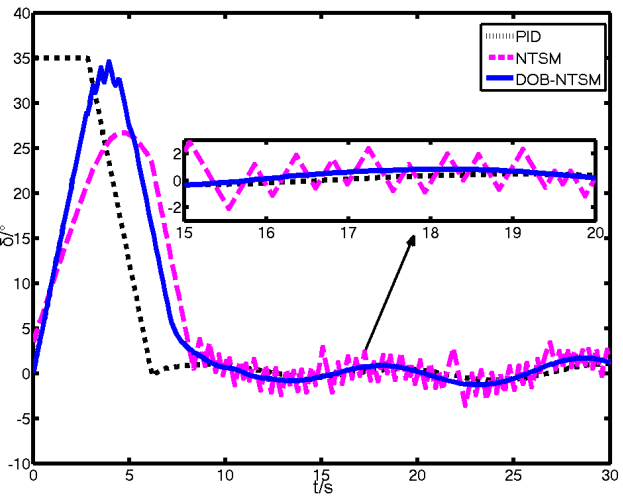

Figure 9. Rudder Angle Curves

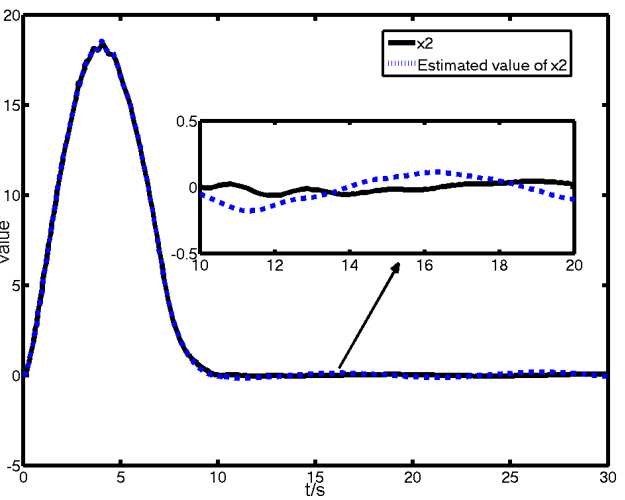

Figure 11. $x_{2}$ Curves

The course control effects of PID, NTSM and DOB-NTSM are shown in Figure 8 from which it is clearly observed that the response speed of the PID controller is the fastest, but in the steady state, the course fluctuates near the target value. At the same time we can see from the local enlargement Fig that the response speed of DOB-NTSM is faster than NTSM, and the steady state performance of NTSM and DOB-NTSM is the same. The rudder angle curves of PID, NTSM and DOB-NTSM are shown in Figure 9 from which it is clearly observed that in the presence of disturbance, the rudder angle of PID and DOBNTSM are very gentle, but the rudder angle of NTSM has a violent chattering. It can be concluded that DOB and RBF have achieved the expected effect.

The disturbance $g(t)$ and its estimated value $\hat{g}(t)$ are shown in Figure 10 from which it is clearly observed that the disturbance observer can track the total disturbance in about 10 seconds. In the subsequent time, the error of $g(t)$ and $\hat{g}(t)$ is very small. The curves of $x_{2}$ and its estimated value $\hat{x}_{2}$ are presented in Figure 11, which shows that the error of $x_{2}$ and $\hat{x}_{2}$ is very small. Further analysis reveals that DOB is effective. In summary, DOBNTSM course controller has better control effect than NTSM controller and PID controller. 


\section{Conclusions}

The object of this research is the Dalian Maritime University "LANXIN" USV. First of all, field experiments are carried out to collect the relevant data, and then system identification method is used to identify the parameters of Norrbin model. Later the simulation experiment is carried out to verify the correctness of the identification results. On the basis of obtaining the model parameters of USV, the NTSM method is used to design the course controller. At the same time, DOB and RBF NN are used to reduce the chattering of the control input. Finally, numerical simulations verify the robustness and feasibility of the DOB-NTSM course controller. In the next step, DOB-NTSM course controller will be applied to "LANXIN" USV to check its control effect.

\section{Acknowledgments}

This work is partially supported by Nature Science Foundation of Liaoning Province of China under Grand 2015020022, Nature Science Foundation of China under Grand 51609033, Fundamental Research Funds for the Central Universities Under Grant 3132014321. The authors also gratefully acknowledge the helpful comments and suggestions of the reviewers, which have improved the presentation.

\section{References}

[1] M. Caccia, M. Bibuli and R. Bono, "Basic navigation, guidance and control of an Unmanned Surface Vehicle", Autonomous Robots, vol. 25, no. 4, (2008), pp. 349-365.

[2] L. P. Perera, P. Oliveira and S. G. Soares, "Dynamic Parameter Estimation of a Nonlinear Vessel Steering Model for Ocean Navigation”, ASME 2011 30th International Conference on Ocean, Offshore and Arctic Engineering. American Society of Mechanical Engineers, (2011), pp. 881-888.

[3] S. Sutulo and C. G. Soares, "An algorithm for offline identification of ship manoeuvring mathematical models from free-running tests", Ocean Engineering, vol. 79, no. 4, (2014), pp. 10-25.

[4] E. R. Herrero and F. J. V. González, "Two-step identification of non-linear manoeuvring models of marine vessels", Ocean Engineering, vol. 53, no. 2, (2012), pp. 72-82.

[5] S. Wirtensohn, H. Wenzl and T. Tietz, "Parameter identification and validation analysis for a small USV", International Conference on Methods and MODELS in Automation and Robotics. IEEE, (2015), pp. 701-706.

[6] C. R. Sonnenburg and C. A. Woolsey, "Modeling, Identification, and Control of an Unmanned Surface Vehicle", Journal of Field Robotics, vol. 30, no. 3, (2013), pp. 371-398.

[7] Y. Zhao, H. Huang and Y. Zhuang, "The Heading Control of POD-Driven Ship Using Adaptive Integrator Backstepping", Proceedings of the 5th International Conference on Electrical Engineering and Automatic Control. Springer Berlin Heidelberg, (2016), pp. 173-179.

[8] G. Xia and T. Luan, "Study of Ship Heading Control using RBF Neural Network", International Journal of Control and Automation, vol. 8, no. 10, (2015), pp. 227-236.

[9] D. Wang and J. Huang, "NN-based adaptive dynamic surface control for a class of uncertain nonlinear systems in strict-feedback form", NNs, IEEE Transactions on, vol. 16, no. 1, (2005), pp. 195-202.

[10] D. Mu, Y. Zhao and G. Wang, "Course control of USV based on fuzzy adaptive guide control”, Control and Decision Conference (CCDC), 2016 Chinese. IEEE, (2016), pp. 6433-6437.

[11] Jia Xinle, Yang Yansheng. Ship motion mathematical model: modeling mechanism modeling and identification. Dalian Maritime University Press, (1999).

[12] System identification and adaptive control of MATLAB simulation, Beijing University of Aeronautics Astronautics Press, (2009).

[13] Feng Y, Yu X, Man Z. Non-singular terminal sliding mode control of rigid manipulators. Automatica, (2002), 38(12): 2159-2167.

[14] Yu X, Man Z. Model reference adaptive control systems with terminal sliding modes. International Journal of Control, (1996), 64(6): 1165-1176.

[15] Man Z, O'Day M, Yu X. A Robust Adaptive Terminal Sliding Mode Control for Rigid Robotic Manipulators. Journal of Intelligent \& Robotic Systems, (1999), 39(1):2464-2469.

[16] Feng Y, Bao S, Xing Y U. Design method of non-singular terminal sliding mode control systems. Control \& Decision, (2002), 17(2):194-198.

[17] Chu Y, Fei J. Adaptive global sliding mode control for MEMS gyroscope using RBF NN. Mathematical Problems in Engineering, (2015), 2015. 
[18] J. Yu, P. Shi and W. Dong, "NN-based adaptive dynamic surface control for permanent magnet synchronous motors", NNs and Learning Systems, IEEE Transactions on, vol. 26, no. 3, (2015), pp. 640-645.

[19] S. Zhang, X. Qiu and C. Liu, "Neural Adaptive Compensation Control for a Class of MIMO Uncertain Nonlinear Systems with Actuator Failures", Circuits, Systems, and Signal Processing, vol. 33, no. 6, (2014), pp. 1971-1984.

\section{Authors}

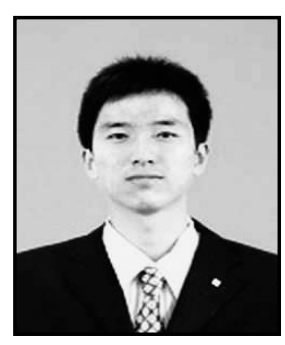

Dongdong Mu, He received his Master's degree in Control theory and Engineering (2015) from Dalian Maritime University. Now he is a PhD Candidate in Dalian Maritime University. His current research interests include USV's model identification and its Non-linear control.

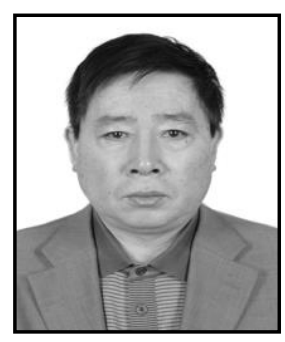

Guofeng Wang, Professor Wang is a doctoral tutor of college of information science and technology, Dalian Maritime University. His current research interests include ship automation and electrical engineering.

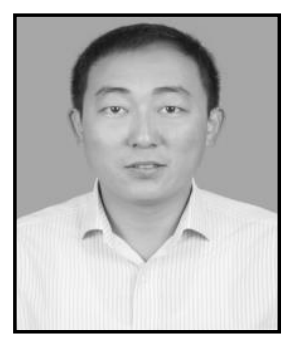

Yunsheng Fan, He received his $\mathrm{PhD}$ in control theory and control engineering (2012) from Dalian Maritime University. His current research interests include ship intelligent control theory and unmanned system measurement and control technology.

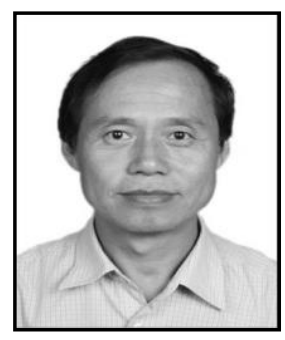

Yongsheng Zhao, Professor Zhao is a master tutor of college of information science and technology, Dalian Maritime University. His current research interests include embedded measurement and control system and computer control. 